\title{
The Nucleus of Active Asteroid 311P/(2013 P5) PANSTARRS
}

\author{
David Jewitt ${ }^{1,2}$, Harold Weaver ${ }^{3}$ (1) Max Mutchler $^{4}$ (1) ${\text { Jing } \text { Li }^{1} \text { (1) , Jessica Agarwal }}^{5}$ (1), and Stephen Larson ${ }^{6}$ (1) \\ ${ }^{1}$ Department of Earth, Planetary and Space Sciences, UCLA, 595 Charles Young Drive East, Los Angeles, CA 90095-1567, USA; jewitt@ucla.edu \\ 2 Department of Physics and Astronomy, UCLA, 430 Portola Plaza, Box 951547, Los Angeles, CA 90095-1547, USA \\ ${ }^{3}$ The Johns Hopkins University Applied Physics Laboratory, 11100 Johns Hopkins Road, Laurel, MD 20723, USA \\ ${ }^{4}$ Space Telescope Science Institute, 3700 San Martin Drive, Baltimore, MD 21218, USA \\ ${ }^{5}$ Max Planck Institute for Solar System Research, Justus-von-Liebig-Weg 3, D-37077 Göttingen, Germany \\ ${ }^{6}$ Lunar and Planetary Laboratory, University of Arizona, 1629 E. University Boulevard, Tucson, AZ 85721-0092, USA \\ Received 2018 March 1; revised 2018 April 6; accepted 2018 April 10; published 2018 May 14
}

\begin{abstract}
The unique inner-belt asteroid 311P/PANSTARRS (formerly P/2013 P5) is notable for its sporadic, comet-like ejection of dust in nine distinct epochs spread over $\sim 250$ days in 2013. This curious behavior has been interpreted as the product of localized, equatorward landsliding from the surface of an asteroid rotating at the brink of instability. We obtained new Hubble Space Telescope observations to directly measure the nucleus and to search for evidence of its rapid rotation. We find a nucleus with mid-light absolute magnitude $H_{V}=19.14 \pm 0.02$, corresponding to an equal-area circle with radius $190 \pm 30 \mathrm{~m}$ (assuming geometric albedo $p_{V}=0.29$ ). However, instead of providing photometric evidence for rapid nucleus rotation, our data set a lower limit to the light-curve period, $P \geqslant 5.4 \mathrm{hr}$. The dominant feature of the light curve is a V-shaped minimum, $\sim 0.3 \mathrm{mag}$ deep, which is suggestive of an eclipsing binary. Under this interpretation, the time-series data are consistent with a secondary/ primary mass ratio, $m_{s} / m_{p} \sim 1: 6$, a ratio of separation/primary radius, $r / r_{p} \sim 4$ and an orbit period $\sim 0.8$ days. These properties lie within the range of other asteroid binaries that are thought to be formed by rotational breakup. While the light-curve period is long, centripetal dust ejection is still possible if one or both components rotate rapidly ( $\lesssim 2 \mathrm{hr}$ ) and have small light-curve variation because of azimuthal symmetry. Indeed, radar observations of asteroids in critical rotation reveal "muffin-shaped" morphologies, which are closely azimuthally symmetric and which show minimal light curves. Our data are consistent with 311P being a close binary in which one or both components rotates near the centripetal limit. The mass loss in 2013 suggests that breakup occurred recently and could even be on-going. A search for fragments that might have been recently ejected beyond the Hill sphere reveals none larger than effective radius $r_{e} \sim 10 \mathrm{~m}$.
\end{abstract}

Key words: comets: general - minor planets, asteroids: general - minor planets, asteroids: individual (311P/ PANSTARRS)

\section{Introduction}

Active asteroids occupy the orbits of asteroids but show the physical appearances of comets, caused by transient mass loss. Their properties indicate a surprising diversity of mass-loss processes, including impact, thermal fracture, and suspected rotational breakup (Jewitt et al. 2015b). One of the strangest such objects is $311 \mathrm{P} / \mathrm{PANSTARRS}$ (formerly $\mathrm{P} / 2013 \mathrm{P}$, hereafter "311P"). Its orbit has semimajor axis, $a=2.189 \mathrm{au}$, eccentricity $e=0.115$, and inclination $i=5^{\circ} .0$, leading to an asteroid-like Tisserand parameter measured relative to Jupiter, $T_{J}=3.662 .311 \mathrm{P}$ orbits near the inner edge of the asteroid belt, where most objects are thought to be highly metamorphized S-types, with suspected meteorite counterparts in the LL chondrites (Keil 2000; Dunn et al. 2013). In addition, the orbital elements of the $10^{9}$ year old Flora asteroid family (Dykhuis et al. 2014) are similar to those of 311P, suggesting that it might be a member. Despite its inner-belt orbit and likely refractory nature, 311P displayed a unique, multi-tailed morphology (Figure 1) caused by episodic dust ejection events (Jewitt et al. 2013, 2015a) spanning the period UT 2013 April 15 (pre-perihelion heliocentric distance $r_{H}=2.304 \mathrm{au}$ ) to December 26 (post-perihelion $r_{H}=1.989 \mathrm{au}$ ). Each tail is a "synchrone" formed by the action of radiation pressure on $\sim 10^{5} \mathrm{~kg}$ of dust launched with initial speeds $<1 \mathrm{~m} \mathrm{~s}^{-1}$. The tails are separated by intervals of inactivity lasting from weeks to months. Jewitt et al. $(2013,2015 \mathrm{a})$ conjectured that 311P might be rotating near the brink of centripetal instability, allowing small avalanches of debris to leave from the equatorial regions and then swept into the observed discrete tails by radiation pressure. This model was quantified and described as "mass-shedding" by Hirabayashi (2015), Hirabayashi et al. (2015), and Scheeres (2015). The importance of rotation in asteroidal mass loss was soon reinforced by observations of another active asteroid, P/2013 R3, in which the body of the asteroid has split into a dozen or more components (Jewitt et al. 2014, 2017).

In this paper, we present new Hubble Space Telescope (HST) observations taken to further examine the development of activity in $311 \mathrm{P}$ and to better characterize the nucleus. The primary objective is to seek evidence for rapid rotation, which is expected if the mass-shedding instability hypothesis is correct.

\section{Hubble Space Telescope Observations}

Observations using the $H S T$ were taken in a series of seven epochs using the Wide Field Camera 3 (WFC3) in imaging mode, allocated under program GO 13866 Table 1). The UVIS channel of the WFC3 camera houses two charge-coupled devices each having $2051 \times 4096$ pixels, with square pixels 0 " 04 on a side. The field of view is $162^{\prime \prime} \times 162^{\prime \prime}$. The image scale projected to the distance of $311 \mathrm{P}$ varied by a factor of two from $38 \mathrm{~km} \mathrm{pixel}^{-1}$ in UT 2015 March to $76 \mathrm{~km} \mathrm{pixel}^{-1}$ in UT 


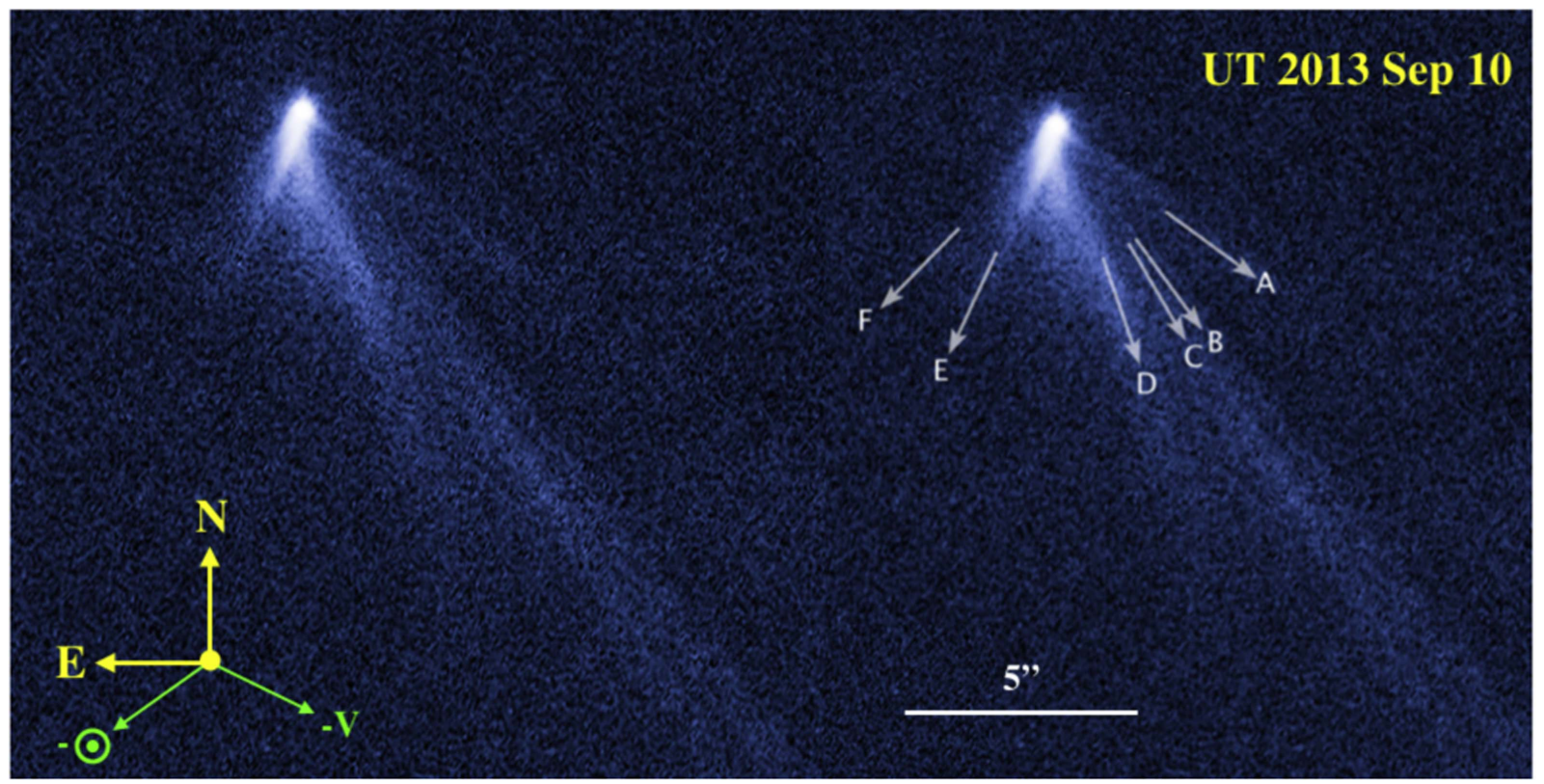

Figure 1. 311P imaged in an active state on UT 2013 September 10 (Jewitt et al. 2013). Letters mark individual dust tails. Three additional tails (G-I) were ejected after this image was taken (Jewitt et al. 2015a). The cardinal directions are indicated by yellow arrows while the projected anti-solar vector $(-\odot)$ and the negative heliocentric velocity vector $(-V)$ are shown in green.

Table 1

Observing Geometry

\begin{tabular}{|c|c|c|c|c|c|c|c|c|c|c|}
\hline UT Date and Time & 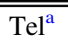 & $\mathrm{DOY}^{\mathrm{b}}$ & 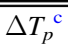 & 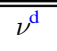 & 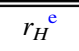 & 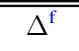 & 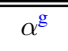 & 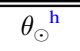 & $\overline{\theta_{-v}{ }^{\mathrm{i}}}$ & 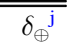 \\
\hline 2014 Nov 17 17:56-18:33 & $H S T$ & 686 & 215 & 78 & 2.111 & 2.280 & 25.7 & 291.6 & 295.6 & -1.5 \\
\hline 2015 Mar 03 14:30-15:06 & $H S T$ & 792 & 321 & 110 & 2.252 & 1.302 & 9.6 & 314.7 & 293.3 & 3.4 \\
\hline 2015 Mar 19 14:05-19:27 & $H S T$ & 808 & 337 & 115 & 2.272 & 1.283 & 3.8 & 21.3 & 294.1 & 3.8 \\
\hline 2015 May 04 06:21-06:58 & $H S T$ & 854 & 383 & 128 & 2.323 & 1.558 & 19.9 & 107.3 & 295.4 & 2.7 \\
\hline 2015 Jun 29 21:03-21:40 & $H S T$ & 910 & 439 & 143 & 2.378 & 2.271 & 25.1 & 114.1 & 294.3 & 0.5 \\
\hline 2015 Jul 27 09:15-09:52 & $H S T$ & 938 & 467 & 150 & 2.399 & 2.616 & 22.8 & 115.1 & 292.9 & -0.8 \\
\hline
\end{tabular}

Notes.

a Telescope used: $H S T=2.4 \mathrm{~m}$ Hubble Space Telescope, $\mathrm{Keck}=10 \mathrm{~m}$ telescope.

${ }^{\mathrm{b}}$ Day of Year, UT 2013 January $01=1$.

${ }^{\mathrm{c}}$ Number of days from perihelion (UT 2014 April $15.78=$ DOY 259).

d True anomaly, in degrees.

${ }^{\mathrm{e}}$ Heliocentric distance, in au.

${ }^{\mathrm{f}}$ Geocentric distance, in au.

${ }^{\mathrm{g}}$ Phase angle, in degrees.

${ }^{\mathrm{h}}$ Position angle of the projected anti-solar direction, in degrees.

${ }^{i}$ Position angle of the projected negative heliocentric velocity vector, in degrees.

${ }^{\mathrm{j}}$ Angle of Earth above the orbital plane, in degrees.

2015 July. We used the F350LP filter, which has a transmission full width at half-maximum $(\mathrm{FWHM})=4758 \AA$ and an effective central wavelength $6230 \AA$ when used to observe a solar-type source.

Five images of 400-420 s duration were taken in each orbit of $H S T$, all significantly contaminated by cosmic rays. We combined the five dithered images using a median filter to reject cosmic rays and detector artifacts, after shifting to align the images on 311P. Magnitudes determined from the orbital median images were measured using a set of concentric photometry apertures. Given the stability of the HST PSF, and with no coma or tail structures evident in the data, we chose to employ very small photometry apertures $(0,2$ and 1 !" 0 radius $)$ in order to minimize uncertainties due to the sky background. We obtained sky subtraction from a concentric annulus having inner and outer radii 1 !" 0 and 4 " 0 , respectively. The photometric calibration assumes that a $V=0$ solar-type source would give a count rate in the F350LP filter of $4.72 \times 10^{10} \mathrm{~s}^{-1}$, as estimated from the on-line exposure time calculator. The average magnitudes within the 0 !! 2 aperture in each HST visit are listed in Table 2. The distribution of the HST observations is shown in Figure 2, where we have also marked and labeled (with red circles) the inferred dates of dust ejections from Jewitt et al. (2013, 2015a).

To measure the magnitudes within individual images, we proceeded as follows. We subtracted the orbital median image from the individual images within the same orbit to remove as far as possible any real signals in the data, leaving only the variable component of $311 \mathrm{P}$, cosmic rays and noise. Then, we removed cosmic rays in the vicinity of the nucleus by hand, 
Table 2

Average Nucleus Photometry

\begin{tabular}{lcccc}
\hline \hline Date & DOY $^{\mathrm{a}}$ & $V_{0.2}{ }^{\mathrm{b}}$ & $H_{0.2}(S)^{\mathrm{c}}$ & $N^{\mathrm{d}}$ \\
\hline 2014 Nov 17 & 686 & $23.47 \pm 0.01$ & $18.92 \pm 0.04$ & 5 \\
2015 Mar 03 & 792 & $22.12 \pm 0.03$ & $19.23 \pm 0.03$ & 5 \\
2015 Mar 19 & 808 & $21.67 \pm 0.03$ & $19.03 \pm 0.03$ & 19 \\
2015 Apr 07 & 827 & $21.98 \pm 0.01$ & $18.95 \pm 0.01$ & 5 \\
2015 May 04 & 854 & $22.71 \pm 0.01$ & $19.04 \pm 0.01$ & 4 \\
2015 Jun 29 & 910 & $23.85 \pm 0.04$ & $19.17 \pm 0.04$ & 5 \\
2015 Jul 27 & 938 & $24.01 \pm 0.01$ & $19.07 \pm 0.01$ & 5 \\
\hline
\end{tabular}

Notes.

${ }^{a}$ Day of Year, UT 2013 January $01=1$.

${ }^{\mathrm{b}}$ Apparent $V$ magnitude within 5 pixel $(0$ !'2) radius aperture. Quoted uncertainty is the statistical error, only.

${ }^{\mathrm{c}}$ Absolute $V$ magnitude, $H_{0.2}$ computed from $V_{0.2}$ assuming an S-type asteroid phase function and Equation (1).

${ }^{\mathrm{d}}$ Number of images used to compute the magnitudes on each date.

replacing them with the average signal from adjacent pixels. Finally, we added back the median image to obtain five cosmicray free images of $311 \mathrm{P}$ per HST orbit. Photometry of these cleaned images was performed as described above. In a few cases, we found images struck by cosmic rays so close to the nucleus of $311 \mathrm{P}$ that no removal was feasible. In these cases, we simply omit the data from further consideration.

\section{Measured Properties}

\subsection{Activity}

The prominent dust tails evident in earlier observations of 311P (Jewitt et al. 2013, 2015a) were absent in the new observations presented here. Figure 3 shows the apparent magnitude for both old and new data as a function of time, expressed as Day of Year (DOY), where DOY $=1$ is defined as UT 2013 January 1. The symbols in the figure represent the averages of measurements taken within a given HST orbit. The associated photometric uncertainties, expressed as the $1 \sigma$ errors on the means, are comparable to or smaller than the sizes of the plot symbols. Also plotted on the figure are curves showing the expected time-variation of the magnitude computed from

$$
V=H+2.5 \log _{10}\left(r_{H}^{2} \Delta^{2}\right)-2.5 \log _{10}(\Phi(\alpha))
$$

where $r_{H}$ and $\Delta$ are the heliocentric and geocentric distances expressed in au, and $\Phi(\alpha) \leqslant 1$ is the phase function at phase angle $\alpha$. The absolute magnitude, $H$, defines the normalization of the curves to the data. It is the apparent magnitude that would be observed if the object were to be located at $r_{H}=\Delta=1$ au and $\alpha=0^{\circ}$. The phase function of $311 \mathrm{P}$ is unmeasured but, over the range of phase angles at which $311 \mathrm{P}$ was observed, is unlikely to have a major effect on the interpretation of the photometry. To show this, we plot in Figure 3 two phase functions representing the nominal behavior of C-type (dashed line) and S-type (solid line) asteroids (characterized by assuming parameter $G=0.15$ and 0.25 , respectively, in the formulation by Bowell et al. 1989). The figure shows that variations in $r_{H}$ and $\Delta$ dominate the longterm photometric variations of $311 \mathrm{P}$ and that the effect of phase angle, $\alpha$, is comparatively modest at the phase angles sampled in our data. In the rest of this work, we assume the phase function of S-type asteroids, guided by the optical color of 311P (Hainaut et al. 2014) and by the location of this object in the inner region of the asteroid belt, where S-types are most abundant.

By fitting the model curves to the data, we observe that most of the early measurements (specifically those from discovery at

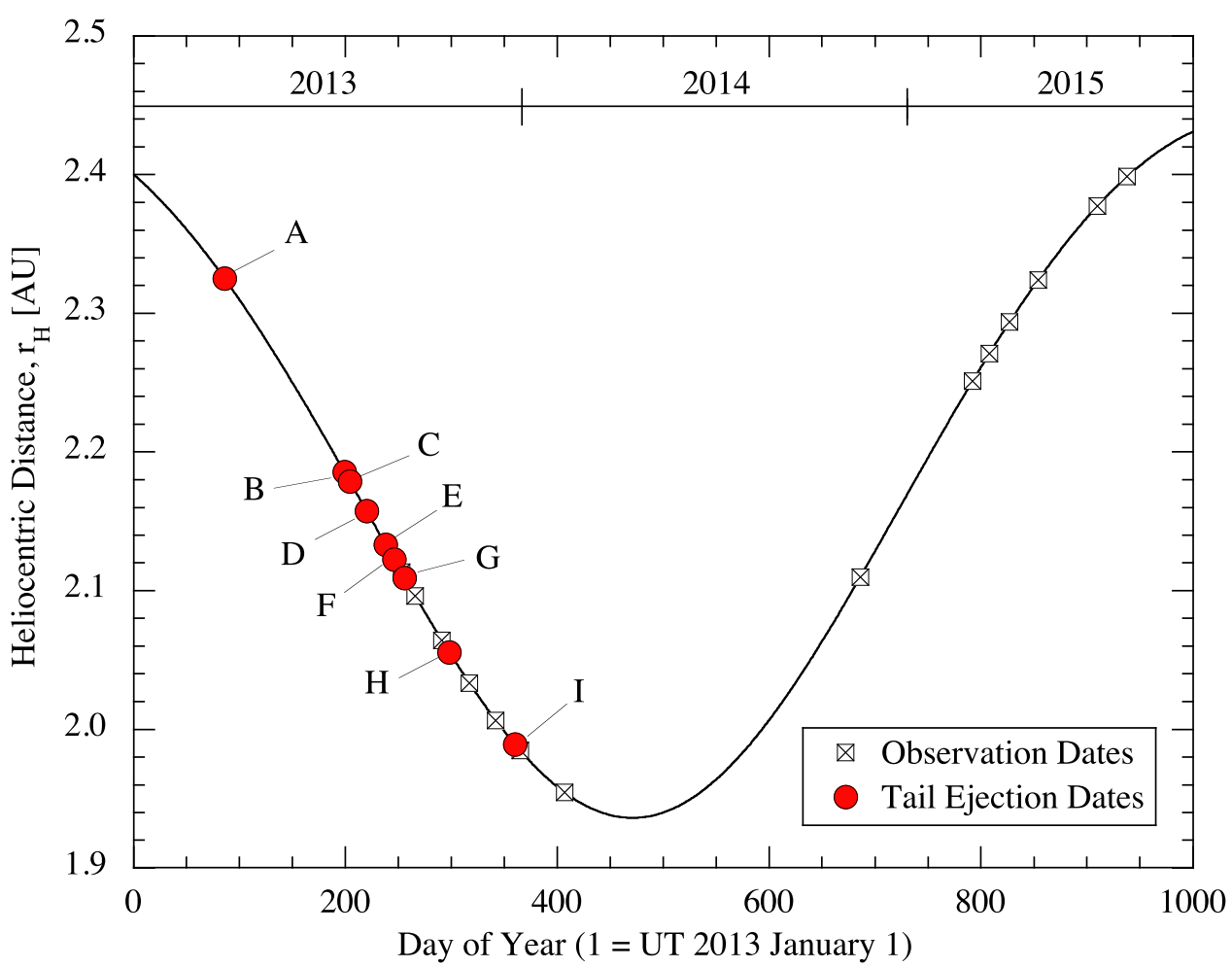

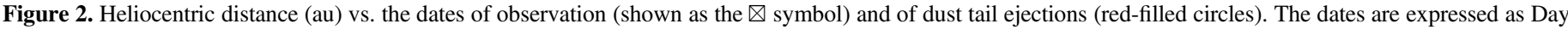

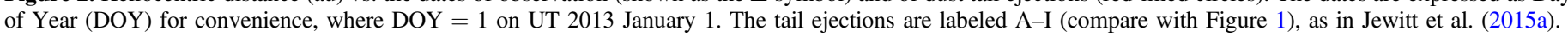




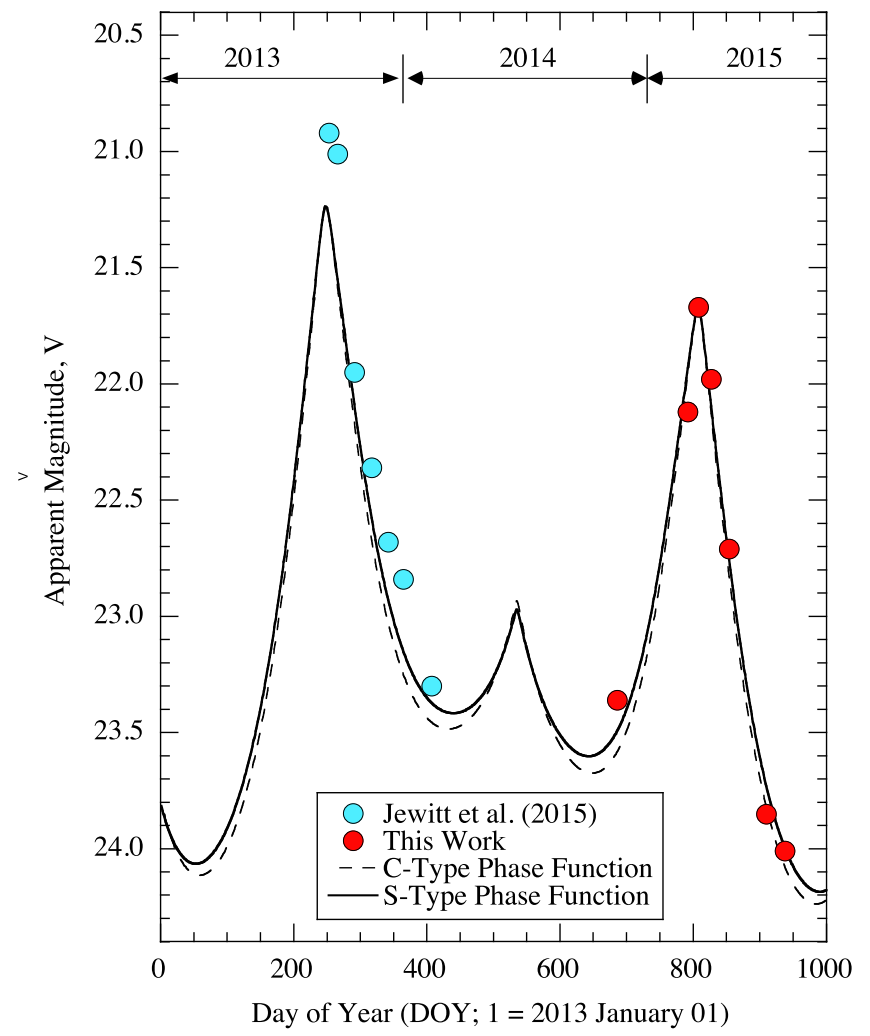

Figure 3. Apparent magnitude measured within a 0 ." 2 radius aperture as a function of time, measured in days, with DOY $=1$ on 2013 January 01. Blue and red symbols distinguish measurements from Jewitt et al. (2015a) from those from Table 2, respectively. Photometric error bars are smaller than the plot symbols. Solid and dashed lines show the model brightnesses for a spherical body following S-type and C-type phase functions, respectively, as described in the text.

$\mathrm{DOY}=230$ to $\mathrm{DOY} \sim 400)$ imply an $H$ systematically brighter than later data (Figure 3). This is clearly seen in Figure 4, where we plot the absolute magnitude as a function of time. We attribute this fact to early dust activity in $311 \mathrm{P}$ and we use only the 2015 photometry from Table 2 to obtain our best estimate of $H$ for the nucleus of 311P. The mid-light value, $H=19.14 \pm 0.02, \quad$ is consistent with a limit, $H \geqslant 18.98 \pm 0.10$, placed from an analysis of data taken in 311P's active phase (Jewitt et al. 2015a). Short-term variations about the mean, in the range $18.95 \leqslant H \leqslant 19.23$, are likely due to light-curve effects.

The absolute magnitude and the scattering cross-section, $C_{e}$, are related by

$$
p_{V} C_{e}=2.25 \times 10^{22} \pi 10^{-0.4\left(H-m_{\odot}\right)}
$$

where $m_{\odot}=-26.75$ is the apparent $V$ magnitude of the Sun (Drilling \& Landolt 2000). We set $p_{V}=0.29 \pm 0.09$, which is the average geometric albedo of the Flora family asteroids as reported by Masiero et al. (2013), to find that $C_{e}$ varies from $C_{\text {min }}=0.10 \mathrm{~km}^{2}$ to $C_{\max }=0.13 \mathrm{~km}^{2}$, with a mid-light value $C_{e}=0.11 \pm 0.04 \mathrm{~km}^{2}$, where the error is dominated by the uncertainty on $p_{V}$. The mid-light cross-section corresponds to the area of a circle of effective radius $r_{e}=\left(C_{e} / \pi\right)^{1 / 2}=190 \pm 30 \mathrm{~m}$ (again, the uncertainty on the effective radius is dominated by the uncertainty on the albedo). This is consistent with an estimate based on earlier HST photometry $\left(r_{e} \leqslant 200 \pm 20 \mathrm{~m}\right.$, Jewitt et al. 2015a) but considerably larger than the radius $15 \leqslant r_{e} \leqslant 65 \mathrm{~m}$ estimated by Moreno et al. (2014) based on a model of the motion of dust. Note that, if our albedo assumption is wrong, both the effective radius and the disagreement with the estimate by Moreno et al. (2014) would likely be larger (e.g., if $p_{V}=0.04$, as for a C-type object, the inferred radius would be larger by the ratio $\left.(0.29 / 0.04)^{1 / 2}=2.7\right)$.

We sought spatially resolved evidence for dust in the data of Table 2 by comparing photometry within concentric apertures. Specifically, we used $\delta V=V_{0.2}-V_{0.4}$ to measure the excess contribution from dust in the $0.2-0$ ". 4 radius range. Light in this annulus includes contributions from the wings of the pointspread function, together with additional light scattered by near-nucleus dust. In all but the UT 2014 November 17 images, the value of $\delta V$ is consistent with expectations based on modeling the point-spread function of HST, for which we used the TinyTim software. Considering all of the data, we find a median $\delta V_{m}=0.092 \mathrm{mag}$. On UT 2014 November 17, we find median $\delta V=0.15 \mathrm{mag}$ and interpret the difference, $\delta V-\delta V_{m}=0.06 \mathrm{mag}$, as caused by light scattered from near-nucleus dust, with an average surface brightness in the 0 " $2-0$ !" 4 annulus of $\Sigma(0$ !" $2-0$ ". 4$)=25.5 \mathrm{mag} \mathrm{arcsec}^{-2}$.

The relation between the surface brightness of a steady-state dust coma measured at radius $\theta^{\prime \prime}$ and the integrated brightness, $V_{\text {Tot }}$, measured within an aperture is (Jewitt \& Danielson 1984)

$$
V_{\mathrm{Tot}}=\Sigma(\theta)-2.5 \log _{10}\left(2 \pi \theta^{2}\right) .
$$

Substituting into Equation (3), we find coma magnitude $V_{\text {Tot }}=26.12$, compared with the measured magnitude of $311 \mathrm{P}$ on UT 2014 November 17 of $V=23.47 \pm 0.01$ (Table 2). If the model coma brightness is subtracted from the measured brightness, we find a bare nucleus magnitude $V=23.57$, about $0.1 \mathrm{mag}$ fainter than in the table. The exact value of the coma correction is uncertain because, for instance, we do not know that the coma is in steady state, as assumed. Steeper (flatter) surface brightness profiles would lead to larger (smaller) coma contributions to the photometry. Nevertheless, it appears that $\sim 10 \%$ of the signal measured on UT 2014 November 17 results from dust while, in all other epochs in Table 2, we find no evidence for coma.

Large particles tend to be ejected slowly and their motions are confined to follow along the projected orbit, forming low surface brightness, nearly parallel-sided "trails" (e.g., Kim et al. 2017). We find no evidence for such a large-particle trail, even in our deepest imaging data (Figure 7). Particles released from the nucleus in 2013 with zero initial velocity would be pushed by radiation pressure to an angular distance $\sim 10^{\prime \prime}$ in our 2015 data if their sizes were $\lesssim 1 \mathrm{~cm}$. The apparent absence of such particles is consistent with the cross-section weighted mean particle size $(3.4 \mathrm{~mm})$ inferred in the tails in earlier images (Jewitt et al. 2015a). Particles of this size would have been dispersed by radiation pressure in the two years since the 2013 outbursts. The low surface brightness excess in the 0 ". 2 to 0 " 4 annulus, described above, is most likely an indicator of small dust grains expelled by late-stage activity. 


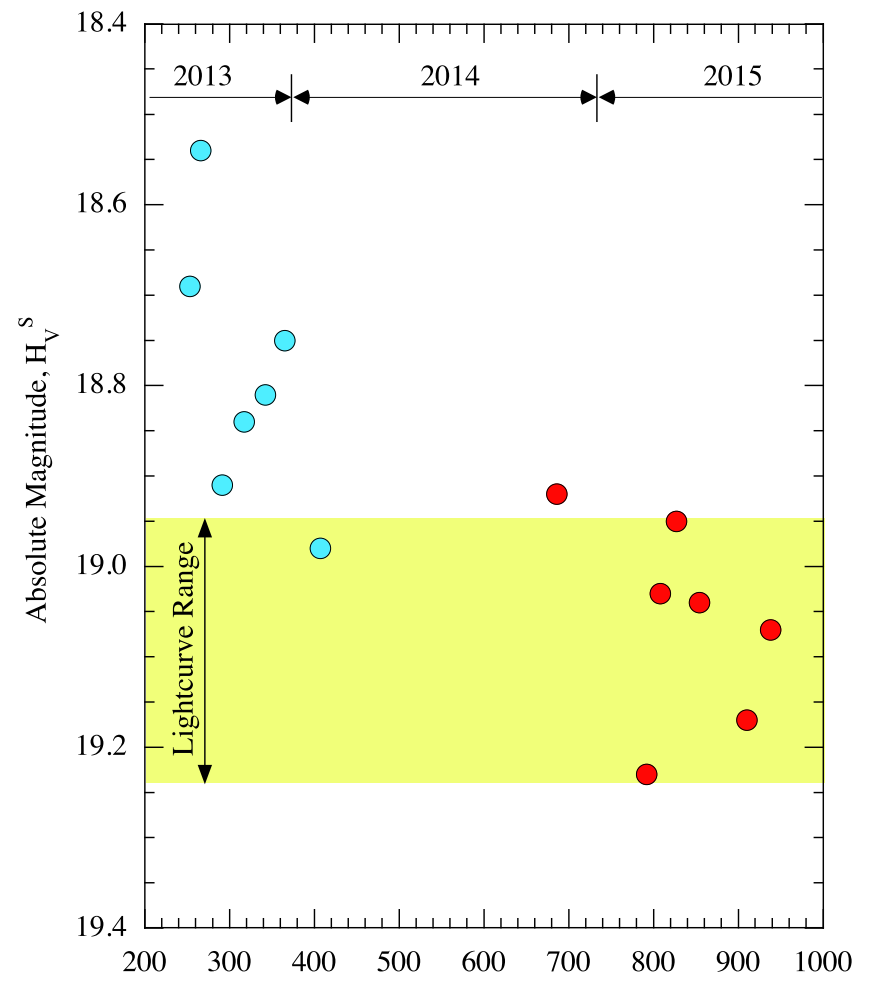

Day of Year $($ DOY $=1=2013$ January 01)

Figure 4. Absolute magnitude measured within a 0.2 radius aperture as a function of time, measured in days, with DOY $=1$ on 2013 January 01. Red and blue circles distinguish the new measurements from this paper from measurements published in Jewitt et al. (2015a), respectively. An S-type phase function has been assumed. The yellow shaded region marks our best estimate of the nucleus light-curve range.

\subsection{Search for Secondary Objects}

A $\sim 190 \mathrm{~m}$ sized body has a very small region of gravitational control, given by the Hill radius,

$$
R_{H}=a\left(\frac{r_{e}}{r_{\odot}}\right)\left(\frac{\rho}{3 \rho_{\odot}}\right)^{1 / 3}
$$

where $a$ is the semimajor axis, $r_{\odot}$ and $\rho_{\odot}$ are the radius and the density of the Sun, respectively, and $r_{e}$ and $\rho$ are again the effective radius and the density of $311 \mathrm{P}$. The latter is unmeasured and so we adopt the mean density of small S-type asteroids, $\rho_{S}=2700 \pm 500 \mathrm{~kg} \mathrm{~m}^{-3}$ as determined by Carry (2012). For comparison, the LL chondrite meteorites are thought to originate from the inner asteroid belt, in the orbital vicinity of $311 \mathrm{P}$. Their average density has been measured at $\rho=3200 \pm 200 \mathrm{~kg} \mathrm{~m}^{-3}$ (Consolmagno et al. 2008). The (small) density difference likely results from the existence of macroporosity in the measured asteroids and from a selection effect whereby denser, stronger asteroid fragments better survive passage through the Earth's atmosphere than do less dense, weaker fragments. We assume that the density of $311 \mathrm{P}$ is well represented by $\rho_{S}$.

Substituting orbital semimajor axis $a=2.189 \mathrm{au}$, $\rho=2700 \mathrm{~kg} \mathrm{~m}^{-3}, \quad \rho_{\odot}=1400 \mathrm{~kg} \mathrm{~m}^{-3}, \quad r_{e}=190 \mathrm{~m} \quad$ and $r_{\odot}=7 \times 10^{8} \mathrm{~m}$, we find $R_{H}=80 \mathrm{~km}$. When observed from minimum distance $\Delta \sim 1.3$ au (as in 2015 March, see Table 1), the Hill radius subtends an angle $\theta_{H}=R_{H} / \Delta \sim 0$ ". 08 , comparable to the $\sim 0$ ". 08 resolution corresponding to Nyquist (2 pixel) sampling of the WFC3 images. Therefore, any existing gravitationally bound companions to $311 \mathrm{P}$ fall beneath the resolution of the $H S T$ data.

In some models of rotational breakup (e.g., Jacobson \& Scheeres 2011; Walsh et al. 2012; Boldrin et al. 2016, discussed later), temporarily stable companion objects are launched from orbit around the primary by complex gravitational interactions, eventually creating asteroid pairs. With radius $r_{e}=190 \mathrm{~m}$ and density $\rho=2700 \mathrm{~kg} \mathrm{~m}^{-3}$, a nonrotating and spherical $311 \mathrm{P}$ would have a gravitational escape speed $V_{e} \sim 0.23 \mathrm{~m} \mathrm{~s}^{-1}$. If traveling at $V_{e}$, an escaping body would spend $4 \times 10^{6} \mathrm{~s}$ (48 days) within $1^{\prime \prime}$ of the primary nucleus and $\sim 2 \times 10^{8}$ s (7 years) within $50^{\prime \prime}$ of it. Objects ejected in 2013, $\sim 400$ days before the present observations, would have traveled $\sim 10^{7} \mathrm{~m}\left(10^{\prime \prime}\right)$ from the nucleus at speed $V_{e}$ and would be well within the WFC3 field of view. Therefore, although we cannot hope to resolve bound companions inside the Hill sphere, it remains worthwhile to search the HST images for evidence of unbound objects that might be slowly leaving the vicinity of the primary.

To this end, we examined the WFC3 images from UT 2015 March 19 (when $\Delta=1.283 \mathrm{au}$ ) in search of co-moving field objects near 311P. We first aligned the images on 311P and then formed image composites using different combinations of the 20 separate images. Each composite image consisted of 10 separate $400 \mathrm{~s}$ exposures, for an effective exposure of $4000 \mathrm{~s}$. Pairs of image composites were then visually compared in order to distinguish spurious sources formed by chance noise clumps and overlapping background trail residuals from potentially real ones. Because of the large parallax motions in these data, background stars and galaxies were not a significant source of confusion except to the extent that they contribute to the background noise. Numerous cosmic-ray tracks in the CCD images were successfully removed in the computation of the composites.

No co-moving objects were found in a region (limited by the position of $311 \mathrm{P}$ on the WFC3 CCD) extending $\sim 40^{\prime \prime}$ to the south, $120^{\prime \prime}$ to the north, and $\sim 80^{\prime \prime}$ east and west of the nucleus. The limiting magnitude of the image composites was estimated in two ways. First, we digitally added sources of known brightness to the data and searched for them in the same way as we searched for real companions. An example grid of digital stars is shown in Figure 5 labeled by their $V$ magnitudes. It may be seen that the visibility of the faintest sources is limited by small variations in "sky" noise caused by the residual images of trailed galaxies. Using the digital stars, we estimate an effective limiting magnitude $V=28.3$, applicable at distances from $311 \mathrm{P}$ greater than $\sim 1^{\prime \prime}$. At smaller separations, the surface brightness from the wings of the point-spread function reduces the sensitivity. Separately, we used the on-line exposure time calculator for the WFC3 camera (http://etc.stsci.edu/) to obtain an independent estimate of the limiting magnitude. For 10 exposures of $400 \mathrm{~s}$ each, on a source with G2V spectral type observed using the 350LP filter, a $3 \sigma$ detection is expected at $V=27.8$. This is slightly poorer than found empirically, a fact which we attribute to our ability to detect objects with formal significance $<3 \sigma$ in blinked-pair data. However, in order to be conservative, we take $V=27.8$ as the upper limit to the allowable brightness of any point-like, co-moving companion.

By Equation (2), $V=27.8$ corresponds to a spherical effective radius $r_{e}=11 \mathrm{~m}$ (again, assuming $p_{V}=0.29$ ) and 


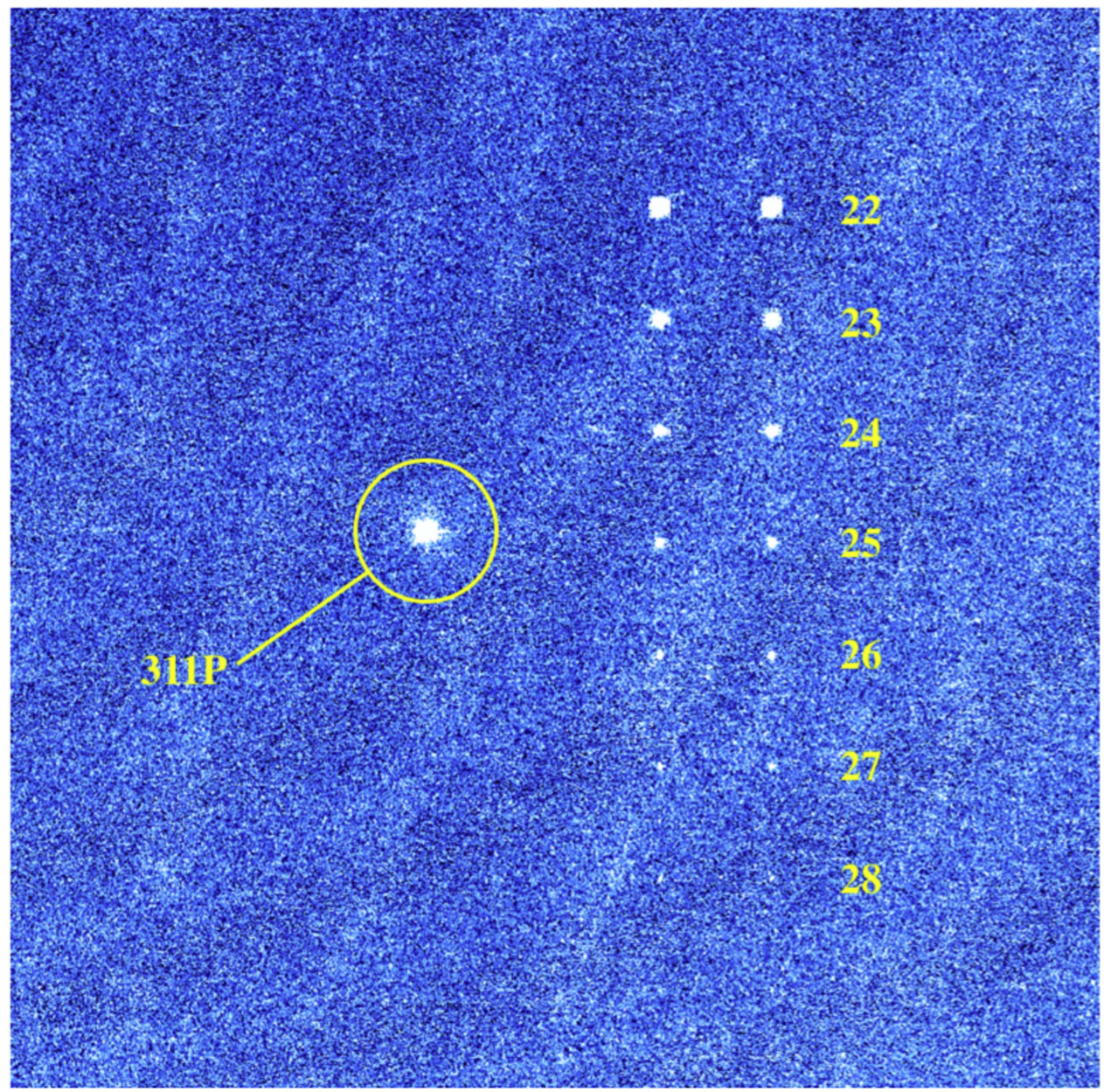

Figure 5. Composite $4000 \mathrm{~s}$ image from UT $2015 \mathrm{March} 19$ showing 311P (circled) and digitally added stars having apparent $V$ magnitudes from 22 to 28 , as labeled. Diagonal streaks in the image are the residuals left by parallactically trailed field galaxies. Width of image shown is $40^{\prime \prime}$.

sets a practical upper limit to the size of any objects ejected from $311 \mathrm{P}$ and still remaining within the vicinity of the nucleus. Such a body would have a mass $\sim 2 \times 10^{-4}$ times that of the central body.

\subsection{Photometric Variations}

Figure 6 shows the individual light curves from the singleorbit visits to $311 \mathrm{P}$. Each visit is limited to $\lesssim 40$ minutes duration by the orbital motion of $H S T$, but even during this short time, the apparent brightness of $311 \mathrm{P}$ is observed to vary by an amount large compared to the \pm 0.01 mag uncertainty of measurement. For example, on UT 2015 March 3 and June 29, the brightness changes by $\sim 0.2 \mathrm{mag}$, or $20 \times$ the measurement uncertainty. The plotted photometry is extracted from a 0 "! 2 radius photometry aperture, corresponding to $\sim 190 \mathrm{~km}$ at $\Delta=1.3 \mathrm{au}$. Within this aperture, the signal is dominated by light from the nucleus and so we presume that most or all of the measured photometric variation is due to rotational modulation of the scattered light, owing to the aspherical shape of the nucleus.

Unfortunately, the majority of the light-curve segments in the figure are so widely separated in time that it is not possible to combine them in order to reconstruct a unique rotational light curve. Instead, the strongest light-curve constraint is provided by the data from four consecutive orbits on UT 2015 March 19. These four orbits span a period of $5.4 \mathrm{hr}$ (Figure 7), and, as the features of the light curve do not repeat, we conclude that a limit to the light-curve period (which is not necessarily equal to the rotation period) may be set at $P_{\ell} \gtrsim 5.4 \mathrm{hr}$.

\section{Discussion}

We briefly consider possible models of the nucleus that conform to this period constraint while providing the centripetal instability inferred to drive intermittent dust loss.

\subsection{Albedo Variations}

If the brightness variations on $311 \mathrm{P}$ are due to azimuthal albedo variations, then the rotational period, $P$, and the lightcurve period are equal, $P=P_{\ell}$. However, azimuthal albedo variations on most small bodies are modest to the point of being undetectable. While we have no specific evidence in the case of 311P, we consider it unlikely that the light curve in Figure 7 is caused by surface albedo variations.

\subsection{Prolate Body}

The light curves of most small bodies in the solar system reflect variations in the projected cross-section caused by rotation. Such geometrically produced light curves are doubly periodic (two maxima and two minima per rotation), meaning 


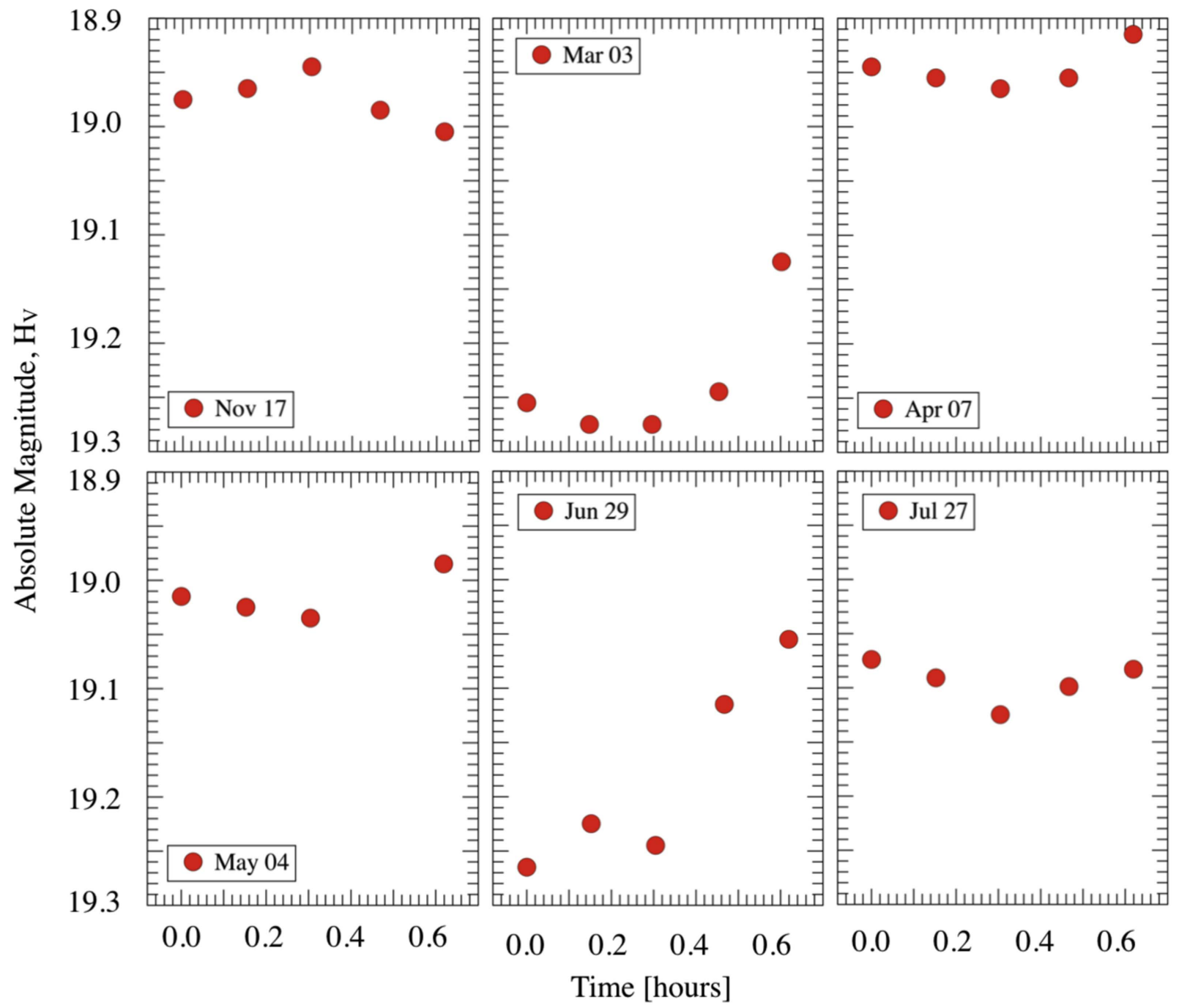

Figure 6. Single-orbit photometry plotted as a function of time in hours for six dates from 2014 November 17 to 2015 July 27 . The absolute magnitude is shown, computed according to Equation (1). Formal error bars, not shown, are comparable to the diameter of the symbols.

that $P=2 P_{\ell}$. In this interpretation, the rotation period of $311 \mathrm{P}$ would be constrained by the data to be $P \geqslant 10.8 \mathrm{hr}$.

The critical period at which a weak body becomes rotationally unstable depends on both its density and its shape. Consider a prolate (American football shaped) body with a long axis of length, $2 b$ and two, equal short axes of lengths $2 a$. In rotation about one of the short axes, the gravity at the tips equals the centripetal acceleration there at critical period

$$
P_{c}=\left(\frac{3 \pi}{G \rho}\right)^{1 / 2}\left(\frac{b}{a}\right) .
$$

The axis ratio, $b / a$, can be estimated from the measured rotational brightness variations, $\Delta V$ using $b / a=10^{0.4 \Delta V}$. Substituting $\Delta V=0.3 \mathrm{mag}$ (Figure 7), we find $b / a=1.3$, and this is formally a lower limit to the axis ratio because of the effects of projection (i.e., the rotation axis might not be perpendicular to the line of sight).

Substituting $\rho=2700 \mathrm{~kg} \mathrm{~m}^{-3}, b / a=1.3$ into Equation (5) we find $P_{c}=2.6 \mathrm{hr}$, which matches the "spin barrier" detected in the rotations of most asteroids larger than $\sim 150 \mathrm{~m}$ in size, Cand S-type alike (e.g., Carbognani 2017, and references therein). Given that $P \gg P_{c}$, we conclude that $311 \mathrm{P}$, interpreted as a single, rotating prolate body, is centripetally stable. Forcing $P_{c}=10.8 \mathrm{hr}$ in Equation (5) gives $\rho<160 \mathrm{~kg} \mathrm{~m}^{-3}$ for centripetal instability, which we consider implausibly small. The light curve (Figure 7) is thus inconsistent with centripetal instability models of the activity in 311P (Jewitt et al. 2013, 2015a; Hirabayashi et al. 2015) if the nucleus is prolate.

\subsection{Oblate Body}

Radar observations (e.g., Ostro et al. 2006; Busch et al. 2011; Naidu et al. 2015) show that many rapidly rotating objects possess an oblate, discus or muffin-like, body-shape likely produced by equatorward migration of material from higher latitudes. A perfectly oblate body in rotation about its minor axis is rotationally symmetric, would have no rotational light curve and so could not be identified using time-series photometry. While radar-observed asteroids are not perfectly oblate, the typical rotational variation in the cross-section is just a few percent (e.g., Pravec et al. 2006), again challenging detection in our data. We cannot appeal to such a body to explain the large photometric variations in 311P. Specifically, the 0.3 mag brightness variation in Figure 7 must have another cause. 


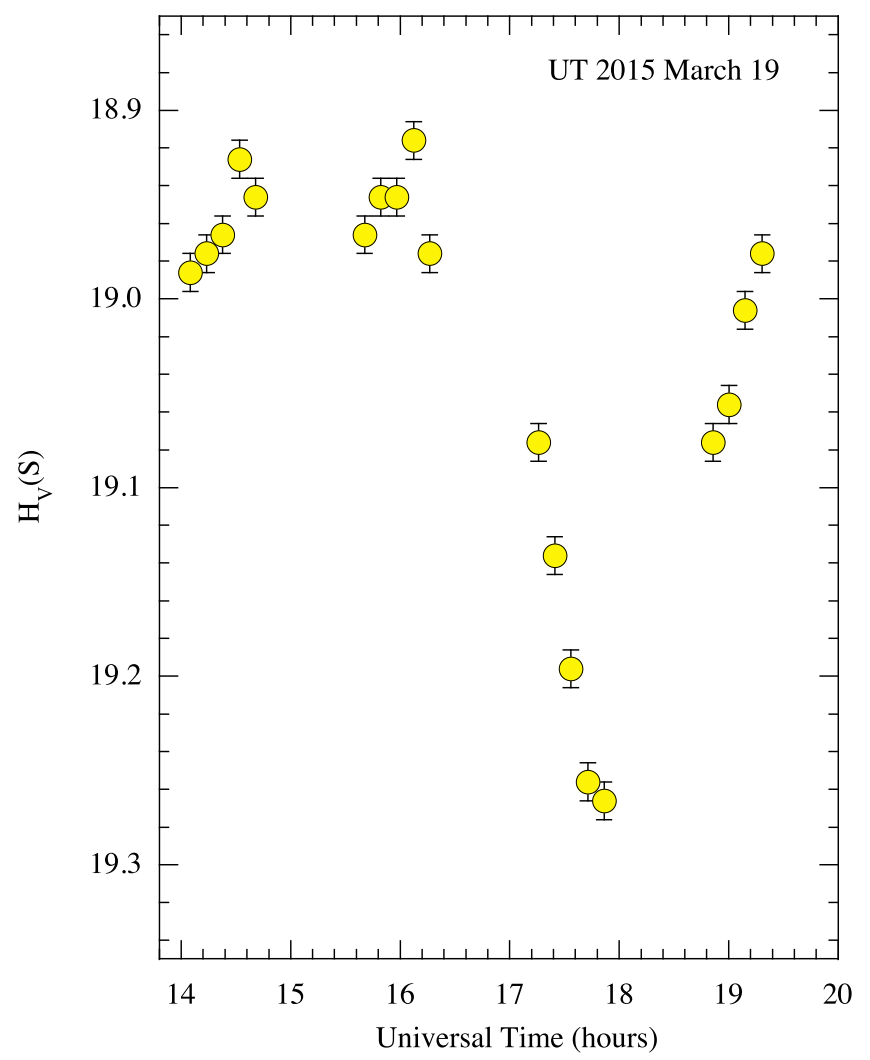

Figure 7. Absolute $V$ magnitude measured within a 0. . 2 radius aperture as a function of time on 2015 March 19. Plotted photometric error bars are $\pm 0.01 \mathrm{mag}$.

With equal long equatorial axes, $2 b$, and short axis, $2 a$, the centripetal and gravitational accelerations are equal in magnitude at the period

$$
P_{c}=\left(\frac{3 \pi}{G \rho}\right)^{1 / 2}\left(\frac{b}{a}\right)^{1 / 2} .
$$

For modest $b / a=1$ to 2 , consistent with the radar-inferred shapes of critically rotating asteroids (e.g., Ostro et al. 2006; Busch et al. 2011; Naidu et al. 2015), and $\rho=2700 \mathrm{~kg} \mathrm{~m}^{-3}$, Equation (6) gives $P_{c}=2.0-2.8 \mathrm{hr}$ for the critical period below which mass could be lost centripetally. This again matches the "spin barrier" detected in the rotations of asteroids (Carbognani 2017) and is substantially smaller than the limit on the period, $P \geqslant 10.8 \mathrm{hr}$.

\subsection{Eclipsing Binary}

The V-shaped drop in the brightness (at rates up to $\sim 0.4$ mag hr ${ }^{-1}$ ) shown in Figure 7 is reminiscent of the light curve of an eclipsing binary (e.g., Pravec et al. 2006, 2016; Lacerda \& Jewitt 2007; Scheirich \& Pravec 2009). In this case, the V-shaped minimum would be caused by the transit or eclipse of a secondary body in an unequal pair. Representing both bodies as spheres having the same albedo and with radii $r_{p}$ (primary) and $r_{s}$ (secondary), we write

$$
C_{\max }=\pi\left(r_{p}^{2}+r_{s}^{2}\right)
$$

at maximum light and

$$
C_{\min }=\pi r_{p}^{2}
$$

at minimum light, assuming a full transit. With $C_{\max }=0.13 \mathrm{~km}^{2}$ and $C_{\min }=0.10 \mathrm{~km}^{2}$, from Section 3.1, we solve Equations (7) and (8) to find $r_{p}=178 \mathrm{~m}$ and $r_{s}=98 \mathrm{~m}$ for the radii of the two components. The mass ratio of these bodies, if they are of the same density, is $m_{s} / m_{p}=\left(r_{s} / r_{p}\right)^{3} \sim 1 / 6$. The observed duration of the supposed transit event, from first contact to last, is $\Delta T=2 \mathrm{hr}$ (Figure 7). From this, we compute the sky-plane velocity of the secondary as it crosses the face of the primary, using

$$
V=2\left(r_{p}+r_{s}\right) / \Delta t,
$$

which gives $V=0.077 \mathrm{~m} \mathrm{~s}^{-1}$. Assuming, for lack of evidence, that the orbit of the secondary is a circle, Kepler's law relates $V$ to the component separation, $r$, through

$$
r=\frac{4 \pi G \rho\left(r_{p}^{3}+r_{s}^{3}\right)}{3 V^{2}}
$$

Substituting, we find $r=840 \mathrm{~m}$ or, in units of the primary radius, $r / r_{p}=4.2$. The separation is a small fraction of the Hill radius (Equation (4)), $r / R_{H}=10^{-2}$. These properties are broadly similar to those of a large number of binary asteroids thought to have been formed by rotational fission (Pravec et al. 2006).

Detection of a transit would only be possible given a specific geometrical alignment of the binary system. Is this alignment likely? In order for a partial transit to be observed, the Earth must lie closer to the orbital plane of the secondary than an angle $\tan (\theta)= \pm\left(r_{p}+r_{s}\right) / r$. We find $\theta= \pm 0.36$ radian $\left( \pm 21^{\circ}\right)$. Given a random distribution of possible orbital poles, the likelihood of finding this alignment by chance is $\sim 62 \%$. To observe a full transit (in which the silhouette of the secondary lies totally within that of the primary) requires $\tan (\theta)= \pm\left(r_{p}-r_{s}\right) / r$, which gives $\theta= \pm 12^{\circ}$. The probability of finding even this more stringent alignment by chance, given a random distribution of planes, is still $\sim 40 \%$. Hence, it is not statistically remarkable that we would detect mutual events given the small separation of this binary.

The orbit period of such a system is $P_{K}=2 \pi r / V$, or $6.8 \times 10^{4} \mathrm{~s}$ (about $19 \mathrm{hr}$ or 0.8 days), meaning that transits and mutual occultations are to be expected every $\sim 9.5 \mathrm{hr}$. There is therefore a high probability that we would detect one minimum in our $5.4 \mathrm{hr}$ observational window. Specifically, given that the $H S T$ observing windows are each $\delta t \sim 40$ minutes $(2 / 3 \mathrm{hr})$ in duration, the probability that a randomly sampled observing window will sample a deep light-curve minimum is $4 \delta t / P_{K} \sim 1 / 7$. In the six single-orbit observing windows shown in Figure 6, we should expect to find $\sim 1$ deep lightcurve minimum. The observations from March 03 definitely record the deep minimum, while observations from June 29 probably just missed it, apparently sampling the rise toward peak brightness.

We conclude that mutual events are quite likely to be detected in a binary with $r / r_{p}=4$ and $P_{K}=19 \mathrm{hr}$, given a randomly selected spin-pole and the temporal sampling of our $H S T$ observations. However, while mutual events in a binary can explain the $0.3 \mathrm{mag}$ dip in the light curve of $311 \mathrm{P}$, they do not provide an explanation for the episodic mass loss indicated by the multiple tails ejected in 2013 (Jewitt et al. 2013, 2015a).

Our suspicion is that the ("landsliding") mechanism indeed drives the mass loss from one or both of the components, but that the rapid rotation required for the instability contributes 


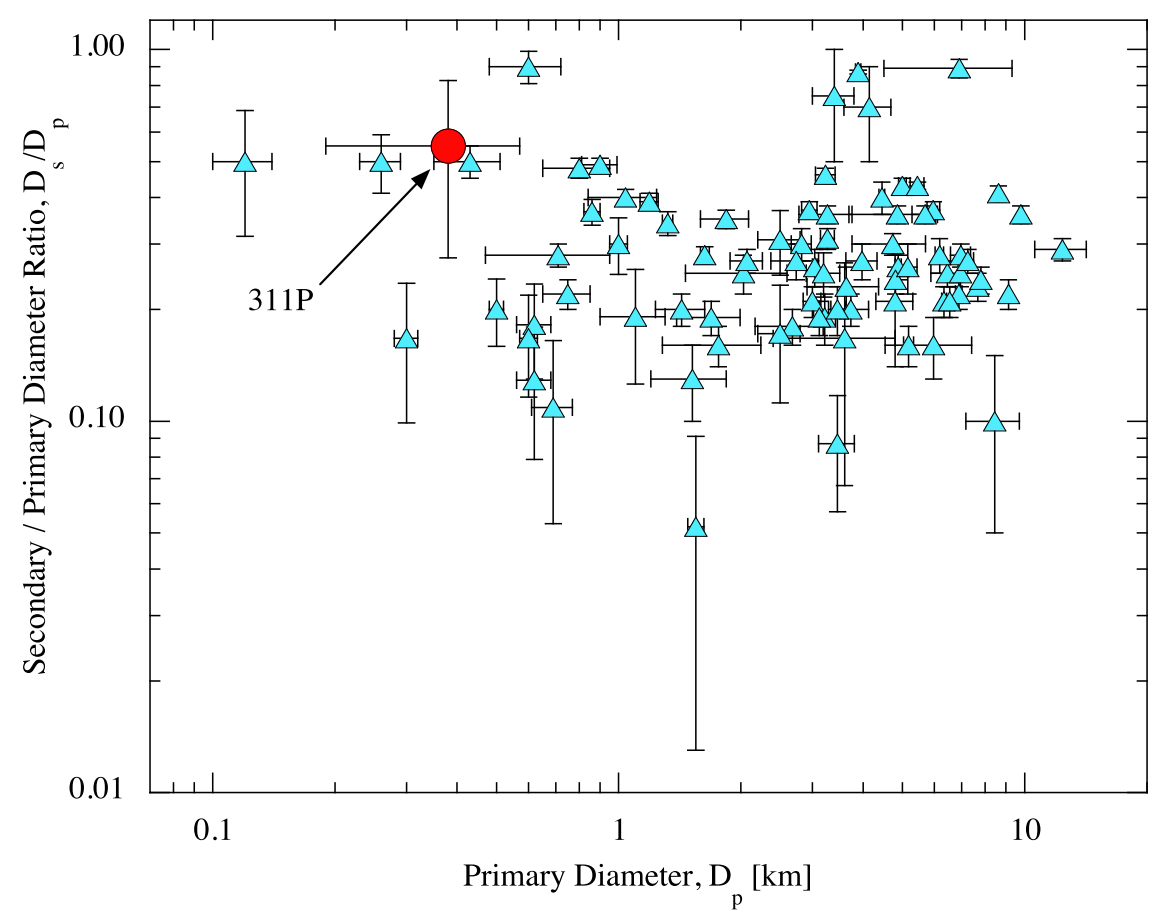

Figure 8. Diameter of the primary component vs. the ratio of secondary to primary component diameters. Asteroid data from the compilation by Johnston (2016) are shown as blue-filled triangles while $311 \mathrm{P}$ is indicated by a red-filled circle.

little to the measured light curves because the unstable body has the rotationally symmetric (muffin-shaped) morphology described above. For example, measurements from individual $H S T$ orbits show short-term variations (Figure 6) that are large compared to the errors of measurement (but small compared to the deep minimum recorded best on UT 2015 March 19; Figure 7). These short-term variations, which we have been unable to phase into a convincing light curve because of the strong aliasing in our widely spaced data, could result from small deviations from rotational symmetry in a critically rotating $(P \sim 2$ hr) muffin-shaped body.

\subsection{Other Models}

Hainaut et al. (2014) conjectured that dust emission from $311 \mathrm{P}$ might be due to friction at the contact point of a "rubbing binary." However, it is not clear, in the context of a rubbing binary, why the interval between dust events should be measured in weeks and months (nine events spread over 250 days; Jewitt et al. 2013, 2015a) rather than being comparable to the orbital period, $P_{K}=19 \mathrm{hr}$. Furthermore, in the absence of a continuous exciting force, and for any plausible values of the friction coefficient or the coefficient of restitution, we expect that relative motion between binary components would be quickly damped-out. These concerns deserve exploration in a future, quantitative treatment of the physics of a binary rubble pile merger.

A final possibility is that $311 \mathrm{P}$ is in an excited rotational state, as a result of its small size and the long damping time due to internal friction. Either or both components of $311 \mathrm{P}$ could be non-principal axis rotators. Excited rotation is common in small asteroids because the damping times are long (Harris 1994), but typically much more data than we possess is required in order to identify the rotational state. Therefore, we must leave this possibility open.

\subsection{Formation}

A leading model for the formation of close asteroid binaries is by the rotational disruption of a weakly cohesive primary (e.g., Walsh et al. 2012; Boldrin et al. 2016; Sánchez \& Scheeres 2016, and see reviews by Margot et al. 2015, Walsh \& Jacobson 2015). Spin-up is driven by radiation torques (the YORP effect) for which the characteristic time at $2 \mathrm{au}$ (estimated from published observations of asteroids in which YORP torque has been detected $)$ is $\tau_{Y}(\mathrm{Myr}) \sim 2\left(r_{p} / 1 \mathrm{~km}\right)^{2}$ (Jewitt et al. 2015b). Beyond the $r_{p}^{2}$ size-dependence in this equation, the YORP timescale is very uncertain for any particular object because it is dependent upon unknown details of the shape, spin vector and surface thermal properties. Nevertheless, for an object as small as $311 \mathrm{P}\left(r_{p} \sim 0.2 \mathrm{~km}\right.$ gives $\left.\tau_{Y} \sim 0.1 \mathrm{Myr}\right)$ it is reasonable to expect that YORP torque has affected the spin. Binaries form when slowly launched debris is captured and reassembled through dissipative collisions in orbit (e.g., Jacobson \& Scheeres 2011; Walsh et al. 2012). The orbits of binaries so-formed are initially chaotic, with some reimpacting the primary and others escaping to infinity (forming asteroid pairs, Pravec et al. 2010; Polishook 2014). Stabilization of the binaries can occur by the transfer of energy from orbital motions into the rotations of the components and by the ejection of mass (and energy and angular momentum) from the system. Jacobson \& Scheeres (2011) found that stable binaries formed only when the mass ratio is $m_{s} / m_{p}<0.2$. This is consistent with our estimate $m_{s} / m_{p} \sim 0.15$ for $311 \mathrm{P}$, but the modeling of disrupting systems is very sensitive to assumptions about the physical properties, most of which are unmeasured, and the agreement may not be significant. A wide binary active asteroid (288P/(300163), with a separation $\left.r / r_{p} \sim 100\right)$ has been discovered but was probably not formed by rotational fission and in-orbit reassembly alone (Agarwal et al. 2017).

We show in Figure 8 a comparison between the inferred secondary/primary object diameter ratio of $311 \mathrm{P}$ with the same 


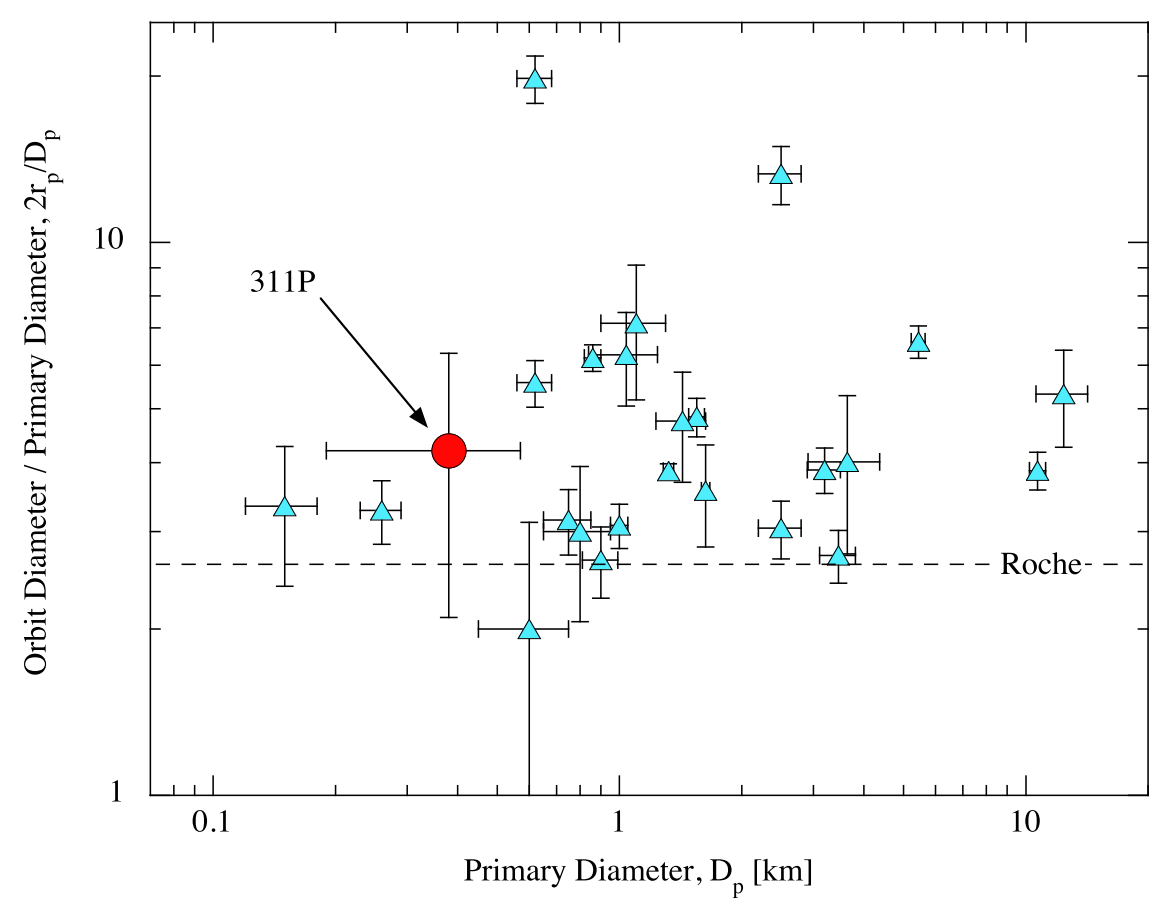

Figure 9. Diameter of the primary component vs. the ratio of the orbit diameter to the primary component diameter. Asteroid data from the compilation by Johnston (2016) are shown as blue-filled triangles while 311P is indicated by a red-filled circle. The dashed horizontal line shows the Roche limit for equal density spheres.

ratio for known binary asteroids having primary diameters $\leqslant 20 \mathrm{~km}$. For the latter, we have used the compilation by Johnston (2016) and retained only those objects for which uncertainties on the primary and secondary object diameters are quoted. The uncertainties on the parameters of 311P are unknown: we show $\pm 50 \%$ errors in diameter and diameter ratio, for illustrative purposes only. While there are relatively few known sub-kilometer binary asteroids, the figure shows that the component diameter ratio of $311 \mathrm{P}$ is not atypical. Similarly, in Figure 9 we compare 311P with the known binaries in the diameter versus orbital radius/primary radius plane, again taking data from Johnston (2016) and plotting only objects $\leqslant 20 \mathrm{~km}$ in diameter for which uncertainties in both diameter and orbit radius/primary radius are quoted. The dashed horizontal line at $r / r_{p}=2.6$ in Figure 9 shows the Roche limiting radius for equal density spheres. It roughly coincides with the lower boundary of the measured binary separations, consistent with the expected very weak nature of secondaries formed by agglomeration in orbit. Again, 311P would be among the smallest binary objects known (this is an artifact of its transient prominence caused by dust ejection) but it is not otherwise remarkable. We conclude that the eclipsing binary interpretation of the 311P light curve is plausible.

Numerical simulations show that the evolution of rotationally disrupted aggregate bodies can be both complex and protracted (Boldrin et al. 2016). Collisions and excitation of the spin of the secondary body can both dissipate orbital energy and so help to stabilize the asteroid as a close binary. The timescales for system evolution can be surprisingly long compared to the orbit period of the system, measured in $100 \mathrm{~s}$ and even 1000s of days. If the detection of 311P in 2013 coincided with its initial breakup, then it seems entirely possible that the dynamical evolution may not yet be finished. The multiple tails, for example, could result from equatorward landsliding on a secondary being spun-up by mutual interactions, as well as from dust escaping from the unstable primary.
Radiation pressure-swept dust, kicked up from the primary by boulders infalling from temporary orbit, could also supply the episodic ejections. Although no departing fragments larger than $\sim 10 \mathrm{~m}$ were detected in our search to date (Section 3.2), it is possible that such objects have yet to be launched and may be detected in future observations. These intriguing possibilities make $311 \mathrm{P}$ an object highly deserving of continued study.

\section{Summary}

We present new observations of $311 \mathrm{P}$ taken to examine the properties of the nucleus in the context of suggestions that sporadic dust release from this body might be caused by rotational instability.

1. We find a mean absolute magnitude $H_{V}=19.14 \pm 0.02$ that, with assumed geometric albedo $p_{V}=0.29 \pm 0.09$, gives a mean effective cross-section $C_{e}=0.11 \pm 0.04 \mathrm{~km}^{2}$. The radius of an equal-area circle is $r_{e}=\left(C_{e} / \pi\right)^{1 / 2}=0.19 \pm 0.03 \mathrm{~km}$.

2. No co-moving secondary bodies brighter than apparent magnitude $V=27.8$ exist beyond $\sim 1^{\prime \prime}$ from the nucleus. This corresponds to an effective radius of $<11 \mathrm{~m}$ (again assuming $p_{V}=0.29$ ).

3. The main features of the light curve are a $\mathrm{V}$-shaped, 0.3 mag deep minimum, and a light-curve period $P>5.4 \mathrm{hr}$. Interpreted as an eclipsing binary, we estimate a secondary/primary radius ratio $r_{s} / r_{p}=0.55$, a circular orbit separation to primary radius ratio $r / r_{p} \sim 4$ (about $10^{-2}$ of the Hill radius), and an orbit period $P_{K} \sim 0.8$ days. These parameters are consistent with those of numerous, small, near-Earth, and main-belt asteroids that are thought to have formed through rotational instability and reaccretion.

4. The observed light curve reflects the orbital motion of the binary but provides no definitive evidence for the rapid rotation inferred to drive centripetal dust loss from 311P. 
However, rotational instability might still be the source of the ejected dust if one or both of the components of the binary have nearly rotationally symmetric shape. Such shapes are expected and observed in radar studies of rapidly rotating asteroids, where they are due to redistribution of particulate matter toward the rotational equator under centripetal accelerations.

We thank the anonymous referee for the helpful comments. Based in part on observations made with the NASA/ESA Hubble Space Telescope, with data obtained via the Space Telescope Science Institute (STSCI). Support for program 13866 was provided by NASA through a grant from STSCI, operated by AURA, Inc., under contract NAS 5-26555. We thank Linda Dressel, Alison Vick, and other members of the STScI ground system team for their expert help.

Facility: HST (WFC3).

\section{ORCID iDs}

Harold Weaver (16 https://orcid.org/0000-0003-0951-7762

Max Mutchler (1) https://orcid.org/0000-0002-0088-3021

Jing Li (ib https://orcid.org/0000-0002-0982-7309

Jessica Agarwal (다 https://orcid.org/0000-0001-6608-1489

Stephen Larson (1) https://orcid.org/0000-0001-6643-6032

\section{References}

Agarwal, J., Jewitt, D., Mutchler, M., Weaver, H., \& Larson, S. 2017, Natur, 549,357

Boldrin, L. A. G., Scheeres, D. J., \& Winter, O. C. 2016, MNRAS, 461, 3982 Bowell, E., Hapke, B., Domingue, D., et al. 1989, Asteroids II (Tucson, AZ: Univ. Arizona Press), 524

Busch, M. W., Ostro, S. J., Benner, L. A. M., et al. 2011, Icar, 212, 649

Carbognani, A. 2017, P\&SS, 147, 1

Carry, B. 2012, P\&SS, 73, 98

Consolmagno, G., Britt, D., \& Macke, R. 2008, ChEG, 68, 1
Drilling, J. S., \& Landolt, A. U. 2000, in Allen's Astrophysical Quantities, ed. A. Cox (New York: AIP, Spinger), 381

Dunn, T. L., Burbine, T. H., Bottke, W. F., \& Clark, J. P. 2013, Icar, 222, 273

Dykhuis, M. J., Molnar, L., Van Kooten, S. J., \& Greenberg, R. 2014, Icar, 243,111

Hainaut, O. R., Boehnhardt, H., Snodgrass, C., et al. 2014, A\&A, 563, A75

Harris, A. W. 1994, Icar, 107, 209

Hirabayashi, M. 2015, MNRAS, 454, 2249

Hirabayashi, M., Sánchez, D. P., \& Scheeres, D. J. 2015, ApJ, 808, 63

Jacobson, S. A., \& Scheeres, D. J. 2011, Icar, 214, 161

Jewitt, D., Agarwal, J., Li, J., et al. 2014, ApJL, 784, L8

Jewitt, D., Agarwal, J., Li, J., et al. 2017, AJ, 153, 223

Jewitt, D., Agarwal, J., Weaver, H., Mutchler, M., \& Larson, S. 2013, ApJL, 778, L21

Jewitt, D., Agarwal, J., Weaver, H., Mutchler, M., \& Larson, S. 2015a, ApJ, 798, 109

Jewitt, D., \& Danielson, G. E. 1984, Icar, 60, 435

Jewitt, D., Hsieh, H., \& Agarwal, J. 2015b, Asteroids IV (Tucson, AZ: Univ. Arizona Press), 221

Johnston, W. R. 2016, NASA Planetary Data System, 244, EAR-A-COMPIL5-BINMP-V9.0

Keil, K. 2000, P\&SS, 48, 887

Kim, Y., Ishiguro, M., Michikami, T., \& Nakamura, A. M. 2017, AJ, 153, 228

Lacerda, P., \& Jewitt, D. C. 2007, AJ, 133, 1393

Margot, J.-L., Pravec, P., Taylor, P., Carry, B., \& Jacobson, S. 2015, in Asteroids IV, ed. P. Michel, F. E. DeMeo, \& W. F. Bottke (Tucson, AZ: Univ. Arizona Press), 355

Masiero, J. R., Mainzer, A. K., Bauer, J. M., et al. 2013, ApJ, 770, 7

Moreno, F., Licandro, J., Álvarez-Iglesias, C., Cabrera-Lavers, A., \& Pozuelos, F. 2014, ApJ, 781, 118

Naidu, S. P., Margot, J. L., Taylor, P. A., et al. 2015, AJ, 150, 54

Ostro, S. J., Margot, J.-L., Benner, L. A. M., et al. 2006, Sci, 314, 1276

Polishook, D. 2014, Icar, 241, 79

Pravec, P., Scheirich, P., Kušnirák, P., et al. 2006, Icar, 181, 63

Pravec, P., Scheirich, P., Kušnirák, P., et al. 2016, Icar, 267, 267

Pravec, P., Vokrouhlický, D., Polishook, D., et al. 2010, Natur, 466, 1085

Sánchez, P., \& Scheeres, D. J. 2016, Icar, 271, 453

Scheeres, D. J. 2015, Icar, 247, 1

Scheirich, P., \& Pravec, P. 2009, Icar, 200, 531

Walsh, K. J., \& Jacobson, S. A. 2015, in Asteroids IV, ed. P. Michel, F. E. DeMeo, \& W. F. Bottke (Tucson, AZ: Univ. Arizona Press), 375

Walsh, K. J., Richardson, D. C., \& Michel, P. 2012, Icar, 220, 514 